\title{
Physical properties of neodymium tin oxide pyrochlore ceramics
}

\author{
Adli A. SAleh ${ }^{1}$, A. F. QAsrawi ${ }^{1,2, *}$, G. YumuşaK ${ }^{3}$, A. Mergen $^{3}$ \\ ${ }^{1}$ Department of Physics, Arab-American University, Jenin, West Bank, Palestine \\ ${ }^{2}$ Group of Physics, Faculty of Engineering, Atilim University, 06836 Ankara, Turkey \\ ${ }^{3}$ Metallurgical and Materials Engineering Dept., Marmara University, 34722 Istanbul, Turkey
}

\begin{abstract}
In this work, physical properties of neodymium tin oxide pyrochlore ceramics prepared by solid state reaction technique are investigated by means of X-ray diffraction, scanning electron microscopy, ultraviolet-visible light (UV-Vis) spectrophotometry and temperature dependent electrical resistivity measurements. The pyrochlore is observed to have a cubic FCC crystal lattice with lattice parameter of $10.578 \AA$. The planes of the cubic cell are best oriented in the [2 22 2] direction. From the X-ray, the UV-Vis spectrophotometry and the electrical resistivity data analysis, the grain size, strain, dislocation density, optical and thermal energy band gaps, localized energy band tail states and resistivity activation energies are determined and discussed. The pyrochlore is observed to have an optical energy band gap of $\sim 3.40 \mathrm{eV}$. This value corresponds to $365 \mathrm{~nm}$ UV light spectra which nominates the neodymium tin oxide pyrochlore ceramics for the use as UV sensors.
\end{abstract}

Keywords: $\mathrm{Nd}_{2} \mathrm{Sn}_{2} \mathrm{O}_{7}$; solid state reaction; $U V$ sensor; optical properties

\section{Introduction}

Owing to its smart features as microwave cavities [1], neodymium tin oxide pyrochlore ceramics has attracted attention of researchers and electronic device designers. In a study of dielectric properties of $\mathrm{Nd}_{2} \mathrm{Sn}_{2} \mathrm{O}_{7}$ ceramics as a circuit element that plays the role of microwave resonator in mobile communication systems, the pyrochlore was reported to have a dielectric constant of 17.02, a quality factor (Qf) of $33,100 \mathrm{GHz}$ and a temperature coefficient of resonant frequency of $-55 \mathrm{ppm} /{ }^{\circ} \mathrm{C}$ when sintered at $1550{ }^{\circ} \mathrm{C}$ for $9 \mathrm{~h}$. On the other hand, well-oriented neodymium doped $\mathrm{SnO}_{2}$ layered nanorod arrays were successfully prepared by substrate-free hydrothermal route technique using sodium stannate and sodium hydroxide. The resulting Nd-doped $\mathrm{SnO}_{2}$ layered nanorod arrays were reported to exhibit a unique nanostructure combined with double layered arrays of nanorods with a diameter of $12 \mathrm{~nm}$ and a length of several hundred nanometers. These nanorods were found to exhibit

*E-mail: atef.qasrawi@atilim.edu.tr excellent features for alcohol gas sensing. It was suggested that the Nd-doped $\mathrm{SnO}_{2}$ nanoarray sensors can be regarded as a promising candidate for detection of alcohol traces in environmental gas monitoring [2]. In addition, high responsivity of neodymium tin oxide pyrochlore to ion irradiation designates it as a promising material for disposal of nuclear waste [3].

Neodymium tin oxide pyrochlore ceramics has been prepared by various techniques including a convenient salt-assisted combustion process using glycine as a fuel to produce a square shaped pyrochlore-type $\mathrm{Nd}_{2} \mathrm{Sn}_{2} \mathrm{O}_{7}$ nanocrystals [4], hydrothermal method to produce low temperature synthesized fine powders of $\mathrm{Nd}_{2} \mathrm{Sn}_{2} \mathrm{O}_{7}$ nanocrystals with a phase-pure pyrochlore [5], dry metallurgical method [6], co-precipitation method [7], sol-gel method [8] and solid state reaction technique (SSR). The advantage of using SSR technique lays in the fact that structural elements can be preserved in the transformation from reactant to product. In addition, the solid reactants react chemically without a presence of any solvent at high temperatures, yielding a stable and pure structured 
material. Moreover, this method is also regarded as an environment friendly since no toxic or unwanted wastes are produced after the solid state reaction is complete. For these reasons, in this work, we are discussing the structural, optical and electrical properties of the neodymium tin oxide pyrochlore ceramics prepared by the conventional high temperature solid state reaction technique. The physical properties connected with the lattice parameters, micro stain, grain size, defect density, optical reflectance and transmittance, energy band gap and band tail states in addition to temperature dependent electrical resistivity are reported in details.

\section{Experimental}

Neodymium tin oxide pyrochlore ceramics with the chemical formula $\mathrm{Nd}_{2} \mathrm{Sn}_{2} \mathrm{O}_{7}$ was prepared by the conventional high temperature solid state reaction technique using a stoichiometric mixture of $\mathrm{Nd}_{2} \mathrm{O}_{3}$ and $\mathrm{SnO}_{2}$. After mixing the powders by ball milling for $6 \mathrm{~h}$ in ethanol using zirconia balls, they were dried and then calcined at $1350{ }^{\circ} \mathrm{C}$ for $10 \mathrm{~h}$. The calcined powders were milled in an agate mortar and then pressed into disks with $\sim 0.15 \mathrm{~cm}$ thickness and $1.0 \mathrm{~cm}$ diameter. The pellets were sintered at $1350{ }^{\circ} \mathrm{C}$ for $4 \mathrm{~h}$, in a tightly closed alumina crucible to prevent evaporation losses. The structure of the samples was investigated using scanning electron microscope JEOL JSM 5600 LV. X-ray diffraction analysis was performed using an $\mathrm{X}$-ray diffractometer (Rigaku, $\mathrm{CuK} \alpha$ radiation) on powdered samples and the unit cell parameters were computed using TREOR 92 software. The electrical contacts to the samples were made from silver paint. The optical measurements were done using Thermo Scientific ${ }^{\mathrm{TM}}$ Evolution $^{\mathrm{TM}} 300$ UV-Vis light spectrophotometer. The electrical resistivity was measured in a homemade cryostat.

\section{Results and discussion}

The X-ray diffraction patterns of the neodymium tin oxide samples sintered at $1350{ }^{\circ} \mathrm{C}$ are displayed in Fig. 1. The figure displays well resolved 8 diffraction patterns with most intensive peak apparent at $29.34^{\circ}$. The peaks were carefully analyzed and compared to the JCPDS Card No. 00-013-0185. All the reflected peaks are observed to relate to a single phase of neodymium tin oxide pyrochlore ceramics. The lattice constant of the unit cell was determined as $10.5730 \AA$. The latter value is very close to that reported as 10.564(6) $\AA$ for the $\mathrm{Zr}$ doped neodymium tin oxide pyrochlore ceramics [9]. Except for one minor peak, all the other reflected peaks relate to the even sum of Miller indices (h k l). The odd sums are absent indicating that it is a face centered cubic structure (FCC).

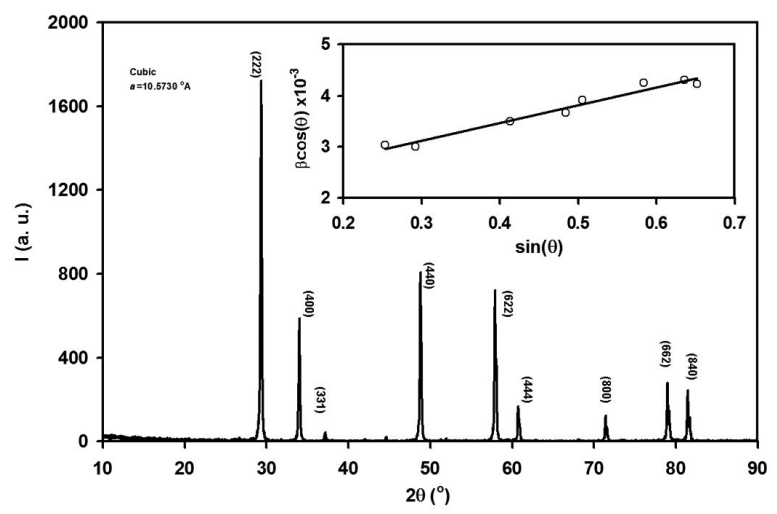

Fig. 1. X-ray diffraction pattern of neodymium tin oxide pyrochlore ceramics. The inset shows the representative plot of $\beta \cos \theta$ versus $\sin \theta$ for the pyrochlore.

The single phase properties of the $\mathrm{Nd}_{2} \mathrm{Sn}_{2} \mathrm{O}_{7}$ pyrochlore have been confirmed by the scanning electron microscopy (SEM) images which are presented in Fig. 2a and Fig. 2b. The image presented in Fig. 2a was recorded at an excitation voltage of $20 \mathrm{kV}$ at a $10^{4}$ magnification. It reflects a very homogeneous crystalline nature of the pyrochlore. The backscattered electrons image which is displayed in Fig. 2b presents a closer look at the crystal structure and shows distribution of grains in pyrochlore. On the other hand, the dispersive energy X-ray (EDXA) qualitative analysis, which is shown in Fig. 2c, does not show any extra impurity in the pyrochlore structure. The apparent $\mathrm{Au}$ peak in the EDXA spectrum is due to the coating of the pyrochlore preventing persistent problems of contamination. 


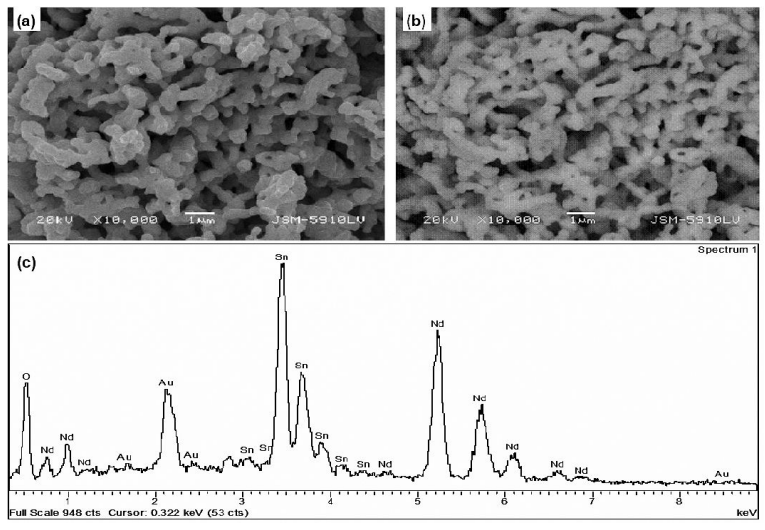

Fig. 2. Representative SEM images of (a) neodymium tin oxide pyrochlore ceramics, (b) backscattered electron image with 10,000 magnification recorded at $20 \mathrm{kV}$ and (c) EDXA spectrum of neodymium tin oxide pyrochlore ceramics.

To explore the mechanical properties of the neodymium tin oxide pyrochlore ceramics, the $\mathrm{X}$-ray diffraction patterns were analyzed by Scherrer approach which is presented by the equation:

$$
\beta \cos \theta=\frac{0.94 . \lambda}{D}+4 \in \sin \theta
$$

where $\epsilon$ is the strain that occurs due to displacement of an unit cell about its normal positions, $D$ is crystallite size and $\beta$ is the peak broadening at full width at half maximum position. The slope and intercept of the of the $\beta \cos \theta-\sin \theta$ function which is shown in the inset of Fig. 1 allowed determining the strain and grain size as $9.95 \times 10^{-4} \mathrm{~nm}$ and $80 \mathrm{~nm}$, respectively. The values of the strain and grain size were used to calculate the dislocation density $\delta$ in the pyrochlore with the help of the equation [10]:

$$
\delta=15 \in /(a D)
$$

The dislocation density was found to be $3.59 \times 10^{14}$ line $/ \mathrm{cm}^{2}$. The determined values of strain, grain size and dislocation density are of importance because they play a significant role in the dielectric relaxation of the pyrochlore ceramics [11]. The existence of the microstrain in this pyrochlore may be ascribed to the large difference between the cation radii of $\mathrm{Nd}^{+3}(143 \mathrm{pm})$ and $\mathrm{Sn}^{+4}$ (69 pm). Such large difference causes lattice distortion and clustering. The later phenomenon was reported for interaction of $\mathrm{Nd}_{2} \mathrm{O}_{3}$ with $\mathrm{CaO}$ and $\mathrm{MgO}$ [12].

The value of the dislocation density being $3.59 \times 10^{14}$ line $/ \mathrm{cm}^{2}$, which is the number of dislocation lines crossing the unit area in the sample is comparable to those reported for other pyrochlore ceramics [10,13]. It plays a vital role in controlling physical properties of the ceramics as it affects the conduction type, radiative recombination and electron lifetime when the pyrochlores are employed for device fabrication [14]. It also remarkably affects the breakdown voltage at which the dielectric ceramic electronic performance, as for example high dislocation density, causes a decrease in crystal density and internal friction, which changes the optical properties and increases the electrical resistance.

The optical reflectivity $\mathrm{R}$ of the neodymium tin oxide pyrochlore ceramics which was recorded in the incident light wavelength range of $190 \mathrm{~nm}$ to $1100 \mathrm{~nm}$ exhibits very low values (less than $1 \%$ ). For this reason the samples were polished with zero level polishing sheets. The maximum obtainable reflectively of the samples was obtained after 500 polishing cycles at a rate of $\sim 50$ cycles $/ \mathrm{min}$. The decrease in the sample thickness $\mathrm{d}$ associated with the 500 polishing cycles was $\sim 20 \%$. The final reflectance spectrum is displayed in Fig. 3a. However, the transmittance spectra were recordable only for the pyrochlore in a powder form with the help of a special band. The band transmission spectra were recorded in the reference compartment to eliminate the contribution of the band to the transmittance spectra of the pyrochlore. The transmission coefficient $\mathrm{T}$ data, which are displayed in Fig. 3a, show a continuously increasing trend with increasing incident wavelength.

The absorption coefficient $\alpha$ which has been calculated from the measured $T$ and $R$ values using the relation:

$$
T=(1-R)^{2} \exp (-\alpha d)
$$

is displayed in Fig. 3b. The absorption coefficient shows an increase with increasing incident light energy. It reaches the maximum absorption level 
at $5.13 \mathrm{eV}$. Further increase in the light energy decreases the absorption coefficient significantly. It is also worth noting that the increase in $\alpha$ value is highly pronounced in the $1.1 \mathrm{eV}$ to $2.2 \mathrm{eV}$ and $3.7 \mathrm{eV}$ to $5.13 \mathrm{eV}$ regions. The plot of the $(\alpha \mathrm{E})^{2}$ vs. E reflects one region of variation that permitted determining the direct allowed transitions energy band gap $\mathrm{E}_{\mathrm{g}}$ of the neodymium tin oxide pyrochlore in accordance with the equation:

$$
(\alpha E)^{2} \propto\left(E-E_{g}\right)
$$

The intersection of $\mathrm{E}$ axis by the extrapolated section of $(\alpha \mathrm{E})^{2}$ plot, which is shown in Fig. 3c, reveals the energy band gap of $3.40 \mathrm{eV}$. This value reflects the sensitivity of the pyrochlore to the ultraviolet light and is comparable to $3.58 \mathrm{eV}$, reported for hexagonal structured $\mathrm{Nd}_{2} \mathrm{O}_{3}[15,16]$ and $3.50 \mathrm{eV}$ for $\mathrm{Nd}_{2} \mathrm{SnO}_{7}$ pyrochlore ceramics [5]. The energy band gap of $\mathrm{Nd}_{2} \mathrm{Sn}_{2} \mathrm{O}_{7}$ ceramics determined from the emission and absorption spectra was assigned to the post-transition between the metal perovskite $\left(\mathrm{ABO}_{3}\right)$ and pyrochlore oxides containing $\mathrm{Sn}^{4+}$. It was also attributed to the electronic interactions with A-site cations mediated through neighboring oxygen atoms [5].
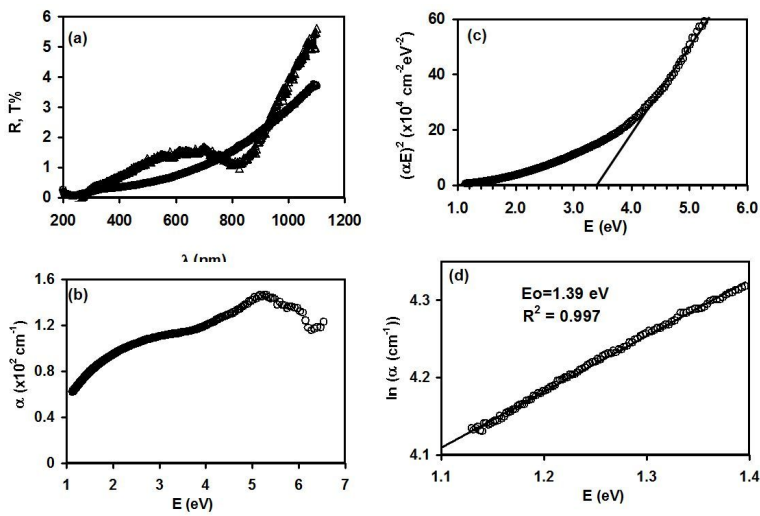

Fig. 3. (a) Optical transmittance and reflectance spectra, (b) absorption coefficient versus light energy, (c) $\left(\alpha^{6} \mathrm{E}\right)^{2}-\mathrm{E}$ and (d) $\ln \alpha-\mathrm{E}$ variations of the neodymium tin oxide ceramics.

In the second region of absorption coefficient from $1.1 \mathrm{eV}$ to $2.2 \mathrm{eV}$, the plot of $\ln \alpha-\mathrm{E}$ reveals a straight line dependence in accordance with $\alpha=\alpha_{0} \exp \left(E / E_{0}\right)$ relation, indicating the possibility of the existence of interbands in the energy band gap of the pyrochlore. The width of the band tails of localized states $E_{0}$ which have been calculated from the slope of the solid line that is presented in Fig. 3d, is found to be $1.39 \mathrm{eV}$. The existence of the band tails is believed to be due to the random fluctuations of the internal fields associated with the structural disorder in the solid pyrochlore ceramics $[16,17]$. The values of the energy band tails in $\mathrm{Nd}_{2} \mathrm{O}_{3}$ and $\mathrm{SnO}_{2}$ were reported to be $\sim 0.20 \mathrm{eV}$ and $1.61 \mathrm{eV}$, respectively. These tails are believed to be extended in respective energy band gaps of $3.51 \mathrm{eV}$ and $3.79 \mathrm{eV}$ [18].

Fig. 4 displays the electrical resistivity of the pyrochlore ceramics registered in the temperature range of $300 \mathrm{~K}$ to $440 \mathrm{~K}$. As it can be observed from the figure, the electrical resistivity is invariant with temperature till the temperature reaches $370 \mathrm{~K}$. Below this temperature, the value of the electrical resistivity is $62.5 \times 10^{9} \Omega \cdot \mathrm{cm}$. When the temperature exceeds $370 \mathrm{~K}$, the electrical resistivity sharply falls with increasing temperature reaching a value of $39.3 \times 10^{7} \Omega \cdot \mathrm{cm}$ at $440 \mathrm{~K}$. The exponential decay of electrical resistivity is observed to follow two regions of variation. In the first region $(370 \mathrm{~K}$ to $400 \mathrm{~K})$, the electrical resistivitytemperature variation reveals activation energy of $1.04 \mathrm{eV}$. In the second region ( $402 \mathrm{~K}$ to $445 \mathrm{~K}$ ), the pyrochlore ceramics exhibits intrinsic properties with thermally activated energy band gap $\left(E_{g}-T h\right)$ of $3.32 \mathrm{eV}$. This value is less than that we optically determined as $3.50 \mathrm{eV}$. The optical gap has been obtained from spectroscopic transitions and thus represents an energy difference between optically determined band edge energies. The optical energies derived from the absorption coefficient can be higher than the minimum difference between the two involved electronic energy levels. In the case of non ignorable electron-hole formation entropy, the thermal gap could be lower than optical gap [19].

\section{Conclusions}

In this article, the structural, compositional, optical and electrical studies of the neodymium tin oxide pyrochlore ceramics have been performed. 


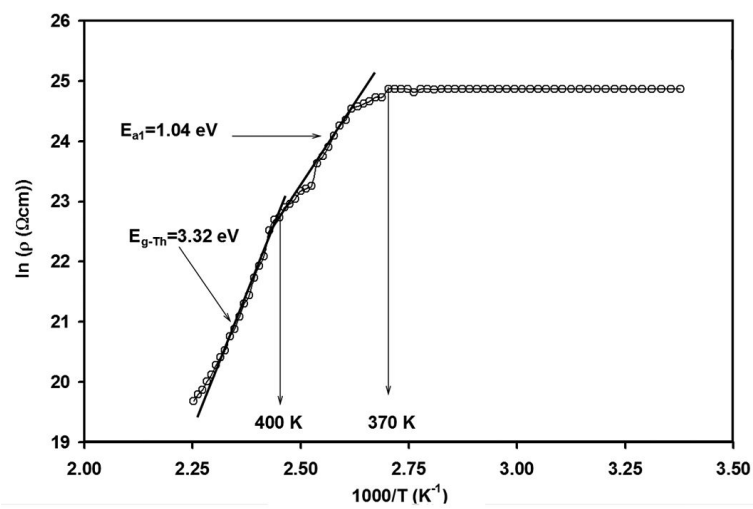

Fig. 4. Variation of electrical resistivity with reciprocal temperature for the neodymium tin oxide pyrochlore ceramics.

The features of the pyrochlore designate it to be effectively used in optoelectronic technology. Namely, the low strain and low dislocation density values as well as nanosize of the grains lead to low lattice mismatch when using this material as an interface in electronic devices. The lattice mismatch is the key problem for the barrier height creation at the device interfaces. In addition, the high absorbability and high energy band gap suggest the applicability of this material in ultraviolet wave sensing. Finally, the optical energy band gap of $3.40 \mathrm{eV}$ and the high temperature stability of electrical resistivity suggests the possibility of using this material in ultraviolet applications up $100{ }^{\circ} \mathrm{C}$.

\section{Acknowledgements}

The authors would like to thank the Scientific Research Council of Arab American University (AAUJ) at Palestine and the Scientific and Technological Research Council of Turkey (TÜBITTAK) for the financial support. The work was supported by the TÜBITAK under the Project FEN-E-120613-0266 and by the AAUJ under the Project Code (2016-2017 Cycle I).

\section{References}

[1] Wang Y., Chen Y., Yao SH., Wu CH., Ceram. Int., 40 (2014), 2641.
[2] Qin G., Gao F., Jiang Q., LiU Y., Li Y., LUO L., Zhao K., Zhao H., Phys. Chem. Chem. Phys. 18 (2016), 5537.

[3] Tracy C. L., Systematic study of the phase behavior of f-block oxides irradiated with swift heavy ions, Ph.D. thesis, Materials Science and Engineering, the University of Michigan (2015).

[4] Tong Y.P., Chen X., Zhao S.B., Lu L.D., Appl. Mech. Mater., 238 (2012), 79.

[5] Alemi A., Kalan R.E., Sci. Plasm. Technol., 163 (2008), 229

[6] Kamel N., Moudir D., Kamel Z., Ait-Amar H., J. Mater. Sci. Eng. B-Adv., 3 (2013), 1.

[7] Feng J., Xiao B., Qu Z.X., Zhou R., Pan W., Appl. Phys. Lett., 99 (2011), 201909.

[8] EWing R.C., Lian J., Wang L.M., MRS Proc., (2003), 792.

[9] Kolekar Y.D., Kulkarni S.B., ChaKraborTHY K., DAS A., PARANJPe S.K., Joshi P.B., Pramana-J. Phys., 63 (2004), 189.

[10] Qasrawi A.F., KMail R.R.N., Mergen A., GenC S.I., J. Electroceram., 37 (2017) 8.

[11] Wang J., Laha A. , Fissel A., Schwendt D., Dargis R., Watahiki T., Shayduk R., Braun W., LIU T., OSTEN J.H., $4^{\text {th }}$ IEEE International Conference on Nano/Micro Engineered and Molecular Systems, 2009, NEMS 2009.

[12] Derouane E.G., Sustainable Strategies for the Upgrading of Natural Gas: Fundamentals, challenges and opportunities, Vol. 191, NATO Science series, Vilamoura, Portogal, 2003, p. 312.

[13] Al Garni S.E., Qasrawi A.F., Mergen A., Ceram. Int., 42 (2016) 3372.

[14] Matsuoka T., Adv. Mater, 8 (1996), 469.

[15] Petit L., Svane A., Szotek Z., Temmerman W.M., Phys. Rev., 72 (2005), 205118.

[16] El-Mallawany R., Abdalla M.D., Ahmed I. A.S, Mater. Chem. Phys., 109 (2008), 291.

[17] Dow J.D., Redfield D., Phys. Rev. B, 5(1972), 594.

[18] Caglara Y., Ilican S., Caglar M., Eur. Phys. J. B, 58 (2007), 251.

[19] Sorrell C. C., Nowotny J., Sugihara S., Materials for Energy Conversion Devices, Woodhead Publishing Limited, England, 2005. 